Tipps \& Tricks im Gyn-Ultraschall

J. Gynäkol. Endokrinol. 2018 · 28:153-157

https://doi.org/10.1007/s41974-018-0075-3

(c) Der/die Autor(en) 2018

CrossMark
Christoph Brezinka

Universitätsklinik für gynäkologische Endokrinologie und Reproduktionsmedizin, Medizinische

Universität Innsbruck, Innsbruck, Österreich

\title{
Sicherheitsaspekte des gynäkologischen Ultraschalls
}

\begin{abstract}
Will man einen Vortragssaal bei einer Ultraschallfortbildung rasch leeren, so kann man „Feuer" rufen. Wenn es nicht brennt, wird der Rufer danach sehr viel Erklärungsbedarf und wahrscheinlich ein Strafverfahren haben. Viel einfacher und genauso schnell geht es mit der Ankündigung „Statt des Vortrags über 3-D-Facing kommt nun ein Beitrag zu den Sicherheitsaspekten des Ultraschalls". Dann werden sich alle im Auditorium erheben und zügigen Schrittes zu den Kaffeeständen der Industrie im Foyer oder zumindest aufs WC gehen. Ich mache mir betreffend dieses Artikels auch keine Illusionen, dass er von der geschätzten Leserschaft nicht zügig überblättert wird. Dennoch möchte ich auf zwei sicherheitsrelevante Themenbereiche hinweisen, wo wir hohe Verantwortung im Alltag des gynäkologischen Ultraschalls und des Ultraschalls in der Frühschwangerschaft haben und durch Unwissenheit und Nachlässigkeit unseren Patientinnen schaden können.
\end{abstract}

\section{Die Schallkopfhygiene}

Der Ablauf ist überall derselbe: Eine Schutzhülle (Kondom) wird mit etwas Schallgel befüllt, über die Vaginalsonde gestülpt, die Ultraschalluntersuchung wird durchgeführt, die Schutzhülle wird entsorgt ... und dann? Wir dürfen nicht vergessen, dass eine Vaginalsonde ein semikritisches Medizinprodukt ist. Das sind Instrumente, die mit Schleimhaut oder krankhaft veränderter Haut in Berührung kommen. „Die alleinige Verwendung einer Latexschutzhülle beim Einsatz einer Vaginalsonde entspricht nicht der erforderlichen Sorgfalt und stellt einen Verstoß gegen den notwendigen Patienten- und Anwenderschutz dar" [1]. Bei alleiniger Verwendung einer Schutzhülle (Kondom) können Schmierinfektionen und Kreuzkontaminationen nicht ausgeschlossen werden. Die Vaginalsonde muss nach jeder Untersuchung einer dezidierten Desinfektionsmaßnahme mit bakterizider, fungizider und viruzider Wirkung unterzogen werden [2]. Dabei darf auch nicht der Griff des Schallkopfs vergessen werden, der ebenfalls ein Risiko für die Übertragung von Keimen darstellt [3]. Wer viele transvaginale Ultraschalluntersuchungen durchführt, wird es immer wieder erleben, dass ein Kondom während der Untersuchung in der Scheide platzt. Damit hat der Schallkopf direkten Kontakt mit Vaginalsekret und oft auch Blut. In so einem Fall muss die Sonde nicht nur sorgfältig gereinigt, sondern gründlich, d.h. auch verlässlich viruzid desinfiziert werden.

Ähnliches gilt auch, wenn die Vaginalsonde zwischendurch als Rektalsonde eingesetzt wird. Für die mancherorts eingerissene Praxis, den Anus im Zuge der Untersuchung als praktischen zweiten Zugang für die Darstellung der Ovarien zu nehmen, sollten jedenfalls klare Hygiene- und Desinfektionsregeln für die dafür verwendeten Schallköpfe festgelegt und befolgt werden. Ein kursorisches Darüberwischen und der Einsatz derselben Sonde fünf Minuten später bei der nächsten Patientin als Vaginalsonde genügt den hygienischen Anforderungen nicht [4].

Die Wahl des Desinfektionsmittels sollte nicht durch die Sparfreudigkeit des Praxisinhabers bzw. des Kranken- hausträgers bestimmt sein, sondern mit den entsprechenden Vorgaben des Herstellers der Ultraschallsonden übereinstimmen: Alkoholhaltige Flüssigkeiten reinigen recht gut, allerdings greifen sie die Schweißnähte der einzelnen Bestandteile der Sonden an, was zu Schäden und einem Erlöschen der Garantie führt. Es müssen dezidiert vom Hersteller für seine Sonden genehmigte Wischtücher verwendet werden, diese enthalten meist organische Ammoniumverbindungen. Grundsätzlich sollte die Reinigung der Ultraschallsonden - einschließlich der Abdominalsonden, die mittlerweile ja auch intrapartal, direkt an der Vulva, zum Einsatz kommen - in einem Protokoll/SOP festgelegt werden, das allen, die mit dem Schallgerät arbeiten, bekannt ist und als verbindlich wahrgenommen wird. Semmelweis' Arbeit über die Keimübertragung beim Kindbettfieber erschien im Jahr 1847 - 172 Jahre später besteht die Verpflichtung zum hygienisch sauberen Arbeiten genauso. Wie damals ist die eigene Nachlässigkeit und Schlamperei immer noch das größte Risiko.

\section{Schallsicherheit - achten auf den Ti!}

Wir sind es von allen Ultraschallmonitoren gewohnt, dass um die eigentliche Bildfläche, auf der das von uns generierte Bild erscheint, eine Reihe von Zahlen und Texten drapiert sind, die dem Ganzen einen technisch-komplizierten Eindruck verleihen. Neben dem Namen der Patientin und dem Datum (die beide stimmen sollten) ist von all diesen Buchstaben- und Zahlenkombinationen nur eine wichtig: der $\mathrm{Ti}$, das ist der „ther- 

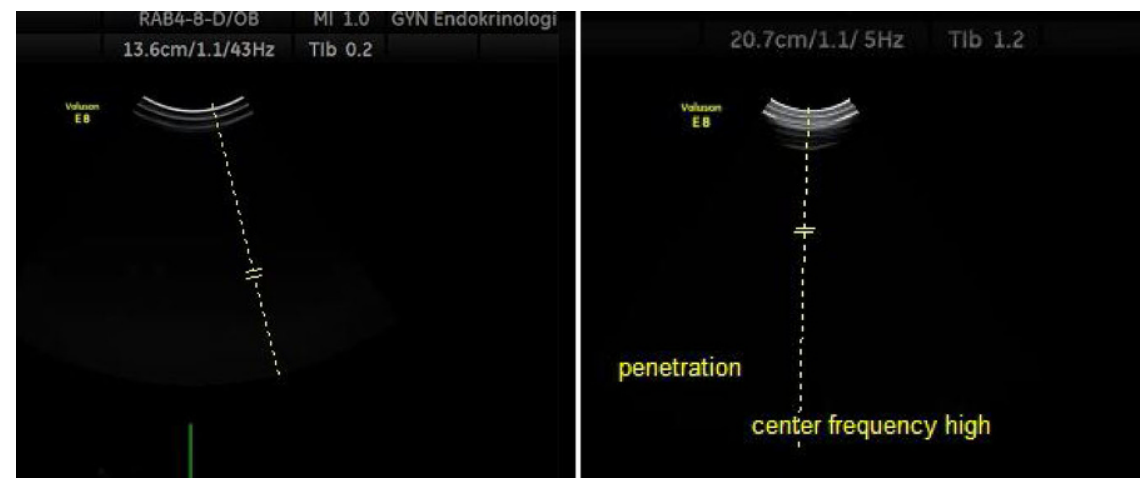

Abb. 1 ॥ Doppler im Ersttrimester-Preset dieses Geräts hat einen Ti von 0,2; schaltet man im selben Gerät auf den Penetration-Preset und "center frequency high", springt der Ti auf 1,2. Während man mit einem Ti von 0,2 eine Stunde schallen kann, halbiert sich bei Ti 1,2 die erlaubte Zeit der Beschallung
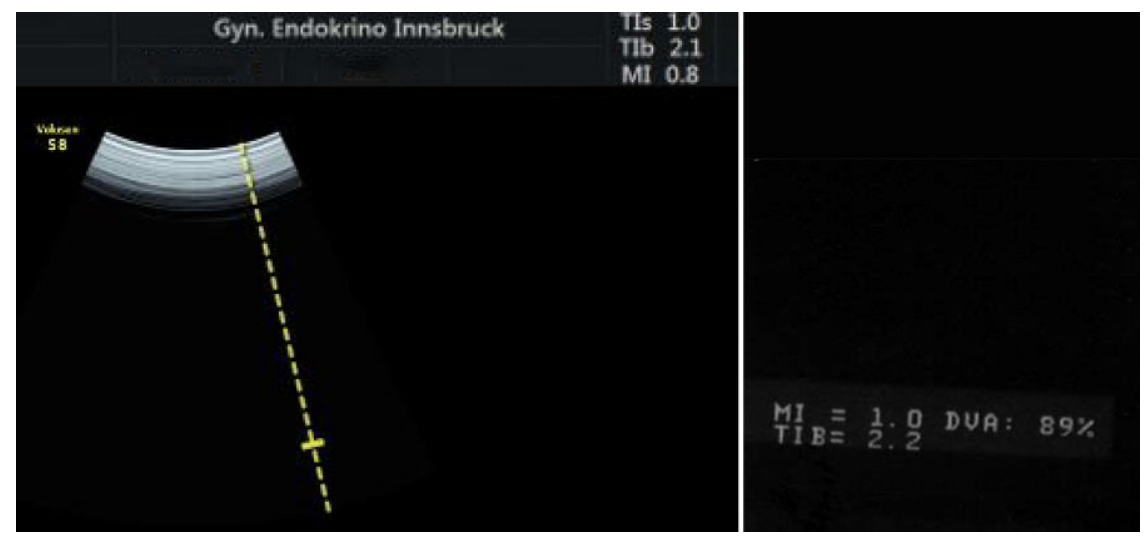

Abb. 2 ॥ Wendet man Doppler außerhalb der „presets“ für das erste Trimester an, kann man problemlos Ti-Werte über 2 erreichen. Bei diesen hohen Werten darfbei einer Schwangeren die Untersuchungsdauer des Dopplers nicht länger als 4 min dauern

mal index“. Dieser sagt aus, um wie viel sich das Gewebe im Wirkungsbereich des Ultraschalls erwärmen kann. Der zweite Index, der „mechanical index“ (Mi) ist für den gynäkologisch-geburtshilflichen Schall bedeutungslos.

Nun wird das Thema der Ultraschallsicherheit im Alltag selten thematisiert, am ehesten noch von Patientinnen (oder ihren Müttern) vom Typus „Lehrerin“. Andererseits gibt es in regelmäßigen Abständen Medienkampagnen über die Gefährlichkeit des Ultraschalls in der Schwangerschaft. Der letzte derartige Versuch war ein Autismusartikel im JAMA Pediatrics im April 2018. Die Autoren erfanden dafür einen neuen Ultraschallindex - ohne ihn zu definieren - „Tiefe der Penetration gewichtet nach Zeit“. Sie sahen sich retrospektiv Bilder in Viewpoint an, maßen von der Hautoberfläche bis zum Kind und den Zeitabstand vom ersten zum letzten Bild der Untersuchung [5].
Bevor wir uns darüber aufregen, wie Leute mit Reizwörtern wie „autism“ im Titel und Phantasieindizes versuchen, Zitationen zu „trollen“, und respektable Journals dem Verhalten durch einen unkritischen Review-Prozess Vorschub leisten, sollten wir uns selbst bei der Nase nehmen und überlegen: Wie viel wissen wir über die Sicherheit des Schalls in der Schwangerschaft [6]? Handeln/schallen wir immer mit maximalem Sicherheitsbewusstsein?

Wer selbst schallt, sollte über die Bioeffekte des Ultraschalls Bescheid wissen, und sei es nur, um die kritischen Fragen der schwangeren Lehrerin beantworten zu können. Die Schallwellen, die von der Sonde in das Gewebe propagiert werden, produzieren Wärme, die im Gewebe absorbiert wird. Das Ausmaß der Temperaturerhöhung nimmt mit der Frequenz, der Expositionsdauer und der „pulse repetition frequency“ (PRF) zu. Man hat einen Industriestandard für die Kalibrierung geschaffen, die „spatial peak temporal average intensity“ (ISPTA). Dies ist die höchste Intensität, die bei einer gegebenen Einstellung an irgendeinem Punkt des Ultraschallstrahls gemittelt über die PRF gemessen werden kann. Sie darf nicht über $720 \mathrm{~mW} / \mathrm{cm}^{2}$ gehen. $I_{S P T A}$ klingt zwar als Parameter wunderschön kompliziert, lässt sich aber während des Schallens nicht messen. Da eine Gefahr für den Embryo vom Ultraschall am ehesten durch eine Erwärmung des Gewebes ausgeht, gibt es den "thermal index“ (Ti). Dieser ist ein eher grobes Maß, das auf der Basis von Messungen mit kalibrierten Hydrophonen und der Schallfrequenz für jedes Schallgerät kalkuliert wird. Ein Ti von 1 entspricht einer Temperaturerhöhung von $1^{\circ}$. Der Index selbst sagt nichts über die Zeitdauer aus, d.h., der Anwender muss selbst entscheiden, wie lange er bei einem bestimmten Ti auf sein Ziel „draufhält“ [7].

Man unterscheidet zwei „thermal indices“: den „Ti soft tissue“ (Tis), dabei geht der Schall nur durch „Weichteile“, da es noch keine knöchernen Strukturen gibt, er wird im ersten Trimenon angewendet. Im zweiten und dritten Trimenon kommt der „Ti bone“ (Tib) zur Anwendung, da nun der Ultraschall auch durch Knochen geht.

Nach den „output display standards“ der FDA müssten alle Ultraschallgeräte, die am Menschen und damit auch an Schwangeren eingesetzt werden, den „thermal index“ an den Monitoren immer deutlich zeigen. Manche Ultraschallhersteller gehen mit dieser Informationsverpflichtung sehr offen und transparent um, andere machen ein regelrechtes Versteckspiel damit. Bei vielen Geräten verschwindet der Ti in dem Moment, in dem man auf die Freeze-Taste drückt - mit der Begründung, dass ein stehendes Bild keine Wärme im Gewebe generiert. Andere lassen den $\mathrm{Ti}$ überhaupt verschwinden und zeigen ihn erst, wenn er über 0,4 ist. Dies hat dann zur Konsequenz, dass die wenigsten Anwender während des Ultraschalls aufden Ti achten. Jeder Anwender ist bemüht, ein möglichst „schönes“ Bild zu generieren, dies geht leider am leichtesten, wenn man mit allen Einstellungen und „presets“ hinauffährt, was dann in 


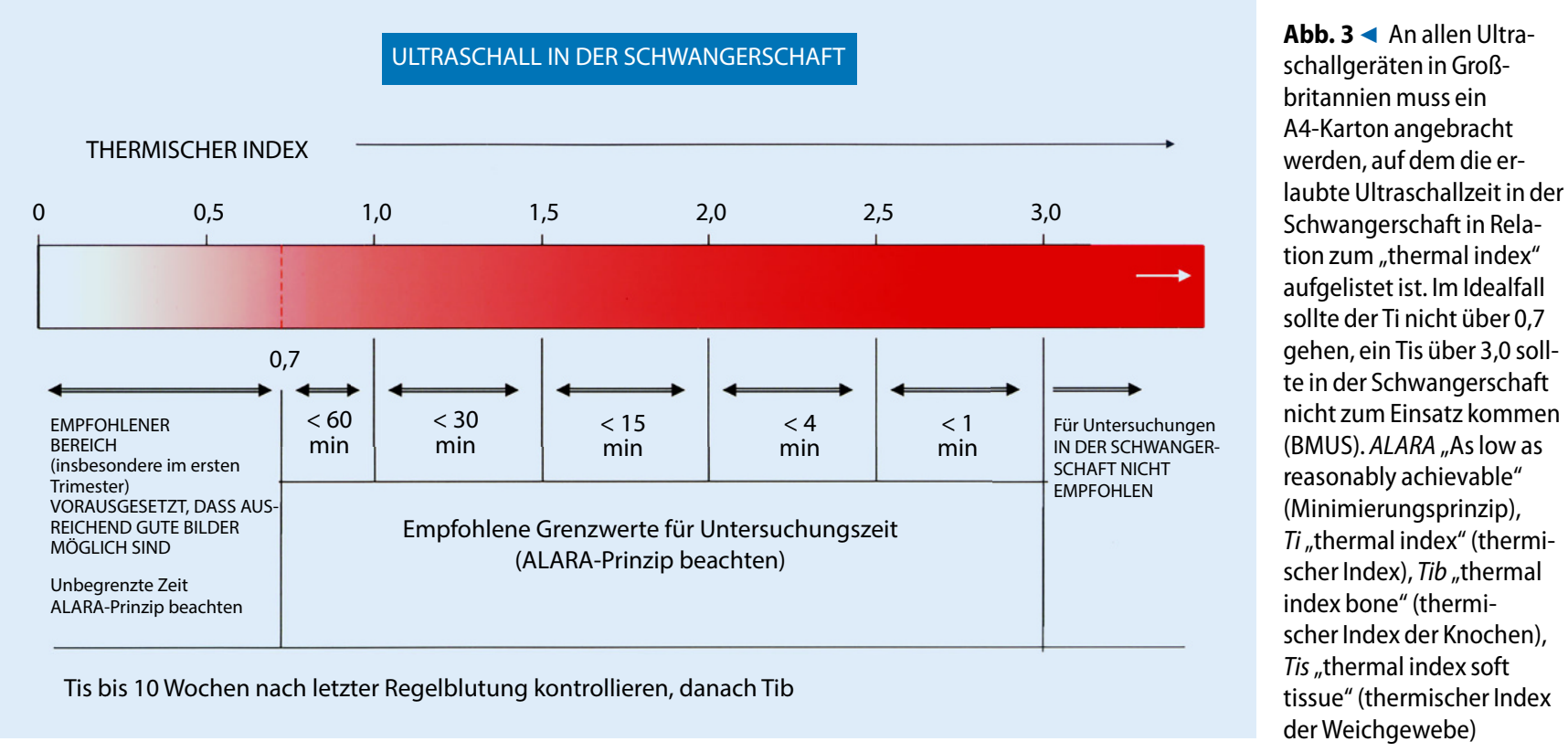

Summe mehr Wärme im beschallten Feld generiert (• Abb. 1, 2 und 3).

Fasst man die wesentlichen Punkte der Ultraschallsicherheit zusammen, so lauten sie:
1. Potenziell gefährlich durch Erhitzung des Gewebes ist nur die Anwendung des Dopplers und hier vor allem in der Frühschwangerschaft.

2. Während der laufenden Ultraschalluntersuchung kann die Intensität des
Schalls anhand des Ti vom Anwender beobachtet werden. Man sollte darauf achten, dass der Ti nicht über 1 geht.

3. Die Praxis des „Herztöne-hörenLassens“, indem man den Doppler über dem $5 \mathrm{~mm}$ großen Embryo

Hier steht eine Anzeige.

\section{帡 Springer}


aktiviert, steht im Widerspruch zu allen Guidelines und Empfehlungen der Ultraschallgesellschaften [8].

4. Versuche, den Dottersack mit Farbdoppler und PW-Doppler zu beschallen, sollten unterbleiben.

5. B-Mode, M-Mode, 3-D- und 4-DUltraschall geben im Gegensatz zum Doppler nur geringe Schallintensitäten weiter und haben daher niedrige Tis.

6. Der Mi ist in der Schwangerschaft von geringer Bedeutung. Er misst die Möglichkeit der Entstehung von Kavitationen, welche im fetalen Schall nicht auftreten können. Er kann außerhalb der Schwangerschaft beim Kontrastmittelultraschall von Bedeutung sein, in der Schwangerschaft zählt nur der $\mathrm{Ti}$ - und der aber wirklich!

Das bewährte Motto „primum nil nocere" gilt auch für die aus dem gynäkologischen Alltag nicht mehr wegzudenkende Anwendung des vaginalen Ultraschalls. Die Keime sind um nichts weniger virulent als zu Semmelweis' Zeiten, allenfalls sind die Antibiotika besser. Wir müssen potenzielle Infektions- und Kontaminationsquellen an unserem Arbeitsplatz im Auge behalten und alles tun, dass keine Patientin den Untersuchungsraum kränker verlässt, als sie ihn betreten hat. Beim Ultraschall in der Frühschwangerschaft weiß zwar niemand genau, ab welchem Grenzwert an Intensität und Zeit es zu einer Schädigung des menschlichen Embryos kommt. Dafür gibt es Sicherheitsgrenzwerte für die Schallexposition, die anhand des $\mathrm{Ti}$ an jedem Monitor mitverfolgt werden können. Prinzipiell gilt, dass Doppler im ersten Trimenon nur überlegt und bei einer klaren Indikationsstellung angewendet werden darf.

\section{Korrespondenzadresse}

Ao. Univ.-Prof. Dr. Christoph Brezinka

Universitätsklinik für gynäkologische

Endokrinologie und Reproduktionsmedizin,

Medizinische Universität Innsbruck

Anichstr. 35, 6020 Innsbruck, Österreich

christoph.brezinka@i-med.ac.at

Funding. Open access funding provided by University of Innsbruck and Medical University of Innsbruck.

\section{Einhaltung ethischer Richtlinien}

Interessenkonflikt. C. Brezinka gibt an, dass kein Interessenkonflikt besteht.

Dieser Beitrag beinhaltet keine vom Autor durchgeführten Studien an Menschen oder Tieren.

Open Access. Dieser Artikel wird unter der Creative Commons Namensnennung 4.0 International Lizenz (http://creativecommons.org/licenses/by/4.0/deed. de) veröffentlicht, welche die Nutzung, Vervielfältigung, Bearbeitung, Verbreitung und Wiedergabe in jeglichem Medium und Format erlaubt, sofern Sie den/die ursprünglichen Autor(en) und die Quelle ordnungsgemäßnennen, einen Link zur Creative Commons Lizenz beifügen und angeben, ob Änderungen vorgenommen wurden.

\section{Literatur}

1. RKI (2018) Aufbereitung von Medizinprodukten. https://www.rki.de/DE/Content/lnfekt/ Krankenhaushygiene/Aufb_MedProd/Aufb_ MedProd node.html.Zugegriffen:Nov. 2018

2. MerzE (2016) Is transducer hygiene sufficient when vaginal probes are used in the clinical routine? Ultraschall Med 37:137-139

3. Buescher DL, Mollers M, Falkenberg MK, Amler S, KippF,Burdach J, KlockenbuschW,SchmitzR(2016) Disinfection of transvaginal ultrasound probes in a clinical setting: comparative performance of automated and manual reprocessing methods. Ultrasound Obstet Gynecol 47:646-651

4. Starostzik C (2017) Keimschleuder Ultraschallsonde. Arztezeitung 13:2

5. Rosman NP, Vassar R, Doros G, DeRosa J, Froman A DiMauro A, Santiago S, Abbott J (2018) Association of prenatal ultrasonography and autism spectrum disorder. JAMA Pediatr 172:336-344

6. ISUOG Safety Committee (2018) Response to JAMA Pediatrics paper on the association of prenatal ultrasonography and autism spectrum disorder by the ISUOG Safety Committee (www.isuog.org)

7. Helmy S, Bader Y, Koch M, Tiringer D, Kollmann C (2015) Measurement of thermal effects of Doppler ultrasound: an in vitro study. PLoSONE 10:e135717

8. Salvesen K, Lees C, Abramowicz J, Brezinka C, ter Haar G, Marsal K (2011) ISUOG statement on the safe use of Doppler in the 11 to $13+6$-week fetal ultrasound examination. Ultrasound Obstet Gynecol 37:628
Toth, B. (Hrsg.)

Fehlgeburten, Totgeburten, Frühgeburten

Ursachen, Prävention und Therapie

Berlin Heidelberg: Springer-Verlag

2017, 1. Auflage, 368 S., 50 Abb., (ISBN: 978-3-662-50423-9), Softcover 54,99 EUR

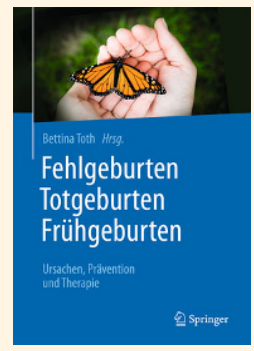

Eine Schwangerschaft zu verlieren stellt ein tragisches Ereignis im Leben einer Frau dar, die sich ein Kind wünscht. Bei wiederholten Schwanger-

schaftsverlusten steigt der psychische Druck immens an. Daher ist sie gewillt alles auf sich zu nehmen, um ein derartiges Ereignis nie wieder erleben zu müssen und wünscht eine aus ihrer Sicht 100\%ige Abklärung der Ursachen. Sie würde alles auf sich nehmen und jegliche Therapie/Prävention durchführen lassen. Daher ist ein Leitfaden zur Betreuung von entsprechenden Patientinnen für betreuende Frauenärzte wünschenswert.

Bei diesem Buch handelt es sich um ein sehr gelungenes Nachschlagewerk, das alle derzeit bekannten Aspekte von Fehl-, Totund Frühgeburten abdeckt und daher dem Leser einen tollen Überblick verschafft. Beschrieben sind nicht nur alle bis dato etablierten Abklärungsmöglichkeiten und therapeutischen Ansätze, sondern darüber hinaus werden auch noch in Forschung befindliche Ursachen diskutiert. Nicht zuletzt wurde bei mehreren Fehlgeburten auch die psychische Belastung aufgegriffen, der eine ganz besondere Bedeutung im Konzept von wiederholten Ereignissen zukommt.

Sehr zu empfehlen ist daher die in diesem Buch systematisch aufgearbeitete Vorgehensweise, im Sinne eines Nachschlagewerks mit zahlreichen Hintergrundinformationen und entsprechenden Referenzen, welches alle beratenden Kollegen, die Frauen mit Fehlgeburten betreuen, in der täglichen Praxis unterstützt.

A. Germeyer (Heidelberg) 
Hier steht eine Anzeige.

\section{曾 Springer}

